\title{
LHOMM L'Homme
}

L'HOMME Revue française d'anthropologie

$194 \mid 2010$

Des maisons et des pierres

\section{Troubles de l'intestin}

Corps et pouvoir dans les Grassfields du Cameroun

Julien Bonhomme

\section{OpenEdition}

Journals

Édition électronique

URL : http://journals.openedition.org/lhomme/22418

DOI : $10.4000 /$ Ihomme.22418

ISSN : 1953-8103

Éditeur

Éditions de l'EHESS

Édition imprimée

Date de publication : 10 mai 2010

Pagination : 157-177

ISSN : 0439-4216

Référence électronique

Julien Bonhomme, «Troubles de l'intestin », L'Homme [En ligne], 194 | 2010, mis en ligne le 05 mai 2012, consulté le 30 avril 2019. URL : http://journals.openedition.org//homme/22418 ; DOI : 10.4000/ Ihomme.22418 


\title{
Troubles de l'intestin
}

\section{Corps et pouvoir dans les Grassfields du Cameroun}

\author{
Julien Bonhomme
}

\begin{abstract}
C
N'EST PAS SEULEMENT parce que les livres de Jean-Pierre Warnier et de Nicolas Argenti portent sur deux sociétés voisines des Grassfields du Cameroun et que les auteurs se connaissent bien et s'entre-citent abondamment qu'ils méritent une analyse croisée. C'est d'abord et avant tout parce que ces deux ouvrages partagent une problématique commune dont l'intérêt anthropologique dépasse largement les particularités ethnographiques de l'aire culturelle abordée : ils s'intéressent à la façon dont la domination est intériorisée par les dominés dans une société hiérarchisée et donc très inégalitaire. Les sociétés des hautes terres de l'Ouest du Cameroun, densément peuplées, s'organisent en effet en une centaine de chefferies de taille très variable. Si les chefferies bamiléké, ou le royaume bamoum, sont les plus connues d'entre elles (Tardits 1960, 1980), les petits royaumes de Mankon et d'Oku dans la partie anglophone des Grassfields sont l'objet des enquêtes de terrain respectives de Jean-Pierre Warnier et Nicolas Argenti. Ces chefferies et royaumes reposent sur la domination du roi (fon) et de l'aristocratie du palais sur les gens du commun. Cette domination politique se traduit par l'exploitation économique des sujets, mais aussi par la polygamie des élites qui contraint une part importante des cadets au célibat à vie. Face à une telle inégalité, comment la royauté peut-elle alors tenir et perdurer? Comment faire accepter la domination aux sujets mêmes qui la subissent? Pour répondre à cette question, Jean-Pierre Warnier et Nicolas Argenti font appel
\end{abstract}

À propos de Jean-Pierre Warnier, Régner au Cameroun. Le Roi-Pot, Paris, Karthala, 2009, et Nicolas Argenti, The Intestines of the State. Youth, Violence, and Belated Histories in the Cameroon Grassfields, Chicago, University of Chicago Press, 2007. 
au concept d' "hégémonie », qui revisite la notion marxiste d' "idéologie » via Gramsci, Foucault, Bourdieu et les Comaroff. Une forme de domination s'exerce de manière hégémonique lorsqu'elle ne se traduit pas par un discours idéologique explicite qui pourrait faire l'objet d'une contestation, mais qu'elle est intériorisée et incorporée par les dominants eux-mêmes au point d'aller de soi et de rester largement implicite (Comaroff \& Comaroff 1991 : 19-32). La domination est en effet d'autant plus efficace qu'elle se passe de mots. Jean-Pierre Warnier et Nicolas Argenti s'intéressent ainsi tout particulièrement aux pratiques non discursives par lesquelles passent les rapports de pouvoir: actions directes sur les corps (Warnier) ou danses de masques (Argenti). Étudier les formes d'intériorisation et d'incorporation de la domination amène alors les auteurs à poser la question de l'adhésion ou de la résistance des dominés. Jusqu'à quel point les cadets du royaume acceptent-ils une domination qui échapperait en partie à l'appréhension consciente? Et dans ces conditions, quelles sont les formes de résistance à leur portée? Sur ces questions, les avis de JeanPierre Warnier et de Nicolas Argenti divergent - et c'est bien là ce qui fait tout l'intérêt d'une analyse croisée de leurs ouvrages.

\section{La gouvernementalité du roi-pot}

Les rapports entre corps et pouvoir font l'objet de longs développements théoriques dans le livre de Jean-Pierre Warnier qui propose en effet un nouveau paradigme en anthropologie politique, creusant un sillon déjà ouvert dans un précédent ouvrage, Matière à politique. Le pouvoir, les corps et les choses (Bayart \& Warnier 2004). S'écartant aussi bien du paradigme sémiologique que socio-fonctionnaliste, cette approche "praxéologique " aborde les rapports de pouvoir à partir de la culture matérielle et sensorimotrice sur laquelle ils s'appuient. Elle procède ainsi d'une critique de ce que Jean-Pierre Warnier appelle l'" effet Magritte" (allusion à Ceci n'est pas une pipe), biais typique de l'illusion scolastique qui confond le monde et sa représentation. Contre un certain logocentrisme de l'anthropologie française, il souligne en effet que le corps n'est pas tant un signe bon à penser qu'un organisme qui agit et sur lequel on peut agir. Jean-Pierre Warnier poursuit ainsi l'héritage maussien des travaux sur les techniques du corps (Mauss 1936), qu'il réactualise par des références au neurobiologiste Alain Berthoz (1997), au sociologue du sport Pierre Parlebas (1999) ou encore à l'anthropologie phénoménologique de l'embodiment (Csordas 1994). Mais c'est surtout l'influence du Michel Foucault de la gouvernementalité, des techniques de pouvoir et de la discipline des corps qui se fait sentir dans cet ouvrage (Foucault 1975, 2008). Jean-Pierre Warnier 
montre ainsi que les relations de pouvoir au principe de la royauté mankon sont "incorporées" dans des techniques matérielles et corporelles. Reprenant la distinction classique entre savoir procédural (savoir en acte) et savoir déclaratif (savoir explicite), il avance que ces techniques de pouvoir reposent sur un savoir procédural qui relève d'un «inconscient moteur et cognitif» et échappe donc en bonne partie à la verbalisation : ce sont des "pratiques qui vont sans dire». Jean-Pierre Warnier transpose ainsi les arguments avancés par Maurice Bloch dans How We Think They Think (1998) pour les appliquer aux relations de pouvoir : une part significative du savoir humain est en réalité non-linguistique. Le savoir ordinaire mobilise en effet des concepts implicites formés dans et par la pratique et ne prend qu'occasionnellement la forme du discours explicite.

S'attachant à mettre au jour les principes de la royauté mankon, JeanPierre Warnier s'inscrit dans le sillage des travaux sur la royauté sacrée en Afrique, thématique classique de l'anthropologie politique depuis James Frazer (Frazer 1981 [1935]; De Heusch 1972, 1982, 2000 ; Adler 1982). Il se démarque cependant des approches structuralistes et fonctionnalistes qui dominent habituellement ce champ de recherches. Il montre que c'est avant tout par son corps que le roi est roi. L'exercice du pouvoir royal dans les Grassfields passe en effet par un imaginaire du corps et des substances : tout corps humain est perçu comme un récipient. Ces corps-récipients se distinguent alors par leurs contenus. Le corps du roi est plein des substances vitales des ancêtres. Celles-ci sont matérialisées dans son souffle, sa salive et son sperme, mais aussi dans le vin de raphia, l'huile de palme et le fard de padouk contenus dans des calebasses qui sont comme les prothèses du corps du roi. Le souverain mankon est ainsi un "roi-pot ", une "tirelire vitale " ${ }^{1}$. Par contraste, les corps de ses sujets sont des récipients vides qui dépendent des substances vitales dispensées par le roi, notamment lors du festival de fin de cycle agricole. Ce dernier a ainsi la charge de "nourrir" et "fortifier" le royaume par le biais de pulvérisations cérémonielles ou encore de la grande polygamie. La royauté s'enracine en somme dans une véritable " physiologie du pouvoir».

Cette conception du corps-récipient se traduit par une attention toute particulière aux enveloppes et à leurs orifices, à ce qui y rentre et ce qui en sort. La peau, signe de santé, reçoit ainsi des soins méticuleux, depuis la toilette du nourrisson jusqu'aux onctions du successeur du roi. Les travaux du psychanalyste Didier Anzieu (1985) ont montré que la peau fait l'objet

1. L'expression "tirelire vitale" n'est pas des Mankon eux-mêmes, mais de prêtres catholiques bamiléké dont la position d'entre-deux leur aurait permis, selon Jean-Pierre Warnier, de mettre en mots ce que les autres ne peuvent habituellement formuler. 
d'un investissement psychique virtuellement universel. Les Grassfields se distinguent alors par un surinvestissement politique des enveloppes corporelles: on passe du «Moi-peau» au "Roi-pot». Une pertinence politique est en effet conférée à un vaste ensemble de pratiques portant sur le corps et la peau, les récipients et les substances. Prolongeant les intuitions séminales d'André-Georges Haudricourt (1962), Jean-Pierre Warnier montre ainsi comment les techniques de gouvernement propres aux Grassfields reposent sur une mise en résonance systématique entre les actions sur la matière et sur les corps d'une part et les actions sur soi et sur autrui d'autre part. La comparaison entre les royaumes des hautes terres et les sociétés de la zone forestière du Sud Cameroun est à cet égard révélatrice. Si ces deux aires culturelles partagent la même attention portée au corps et aux substances, elles s'opposent en revanche sur les techniques de pouvoir étayées sur ces pratiques. Tandis que dans les hautes terres, les substances circulent verticalement du roi vers ses sujets, dans le Sud Cameroun, elles circulent horizontalement entre tous les hommes, dès lors qu'ils sont initiés. Cette différence se traduira dans des pratiques très concrètes, comme le passage de main en main d'une calebasse de vin de palme. Les sociétés lignagères de la forêt se caractérisent en effet par une idéologie égalitaire à l'opposé de la stratification sociale des chefferies des hautes terres (Laburthe-Tolra 1985). C'est pourquoi on n'y trouve pas de clôture hiérarchique sur le corps du roi.

Le corps du roi est mis en relation fractale avec une série d'autres récipients selon un principe typiquement analogiste (au sens de Philippe Descola, 2005). En effet, un système politique comme la royauté sacrée suppose nécessairement une cosmologie analogiste qui ordonne les êtres selon une chaîne hiérarchique dont le roi est le sommet. Le royaume mankon se définit par une série d'emboîtements, à l'image des poupées russes. Les calebasses contenant le fard royal, le palais du souverain, la cité de Mankon sont autant de répliques du corps du roi, irriguées par ses substances vitales. Nicolas Argenti note ainsi à propos de la chefferie d'Oku que le palais représente les «intestins de l'État». En ce sens, les royautés des Grassfields illustrent exemplairement la notion de corporate group, chère à l'anthropologie britannique : le collectif humain est pensé comme un organisme à l'image du corps du roi. À partir d'une comparaison avec la théorie des «deux corps du roi » dans la théologie politique médiévale (Kantorowicz 1989), Jean-Pierre Warnier montre comment la succession royale constitue alors un événement particulièrement sensible : le corps de l'héritier doit être refaçonné en un corps politique englobant qui lui permet d'"avaler» le royaume. Du fait de cet analogisme fractal, on retrouve la même attention aux limites de l'enveloppe corporelle à 
une échelle agrandie: seuil des maisons, enceinte du palais, tranchée entourant la cité. La circulation des biens et des êtres est alors perçue comme un vaste mouvement d'incorporation au royaume: le corps de la cité absorbe des biens, accaparés par les notables du palais, mais aussi des femmes, elles aussi monopolisées par l'aristocratie polygame, ainsi que des cadets en rupture de ban avec leur chefferie d'origine, qui viennent se mettre au service de nouveaux maîtres. À l'inverse, la cité expulse chaque année des sorciers à l'occasion d'une cérémonie d'ordalie du poison : ces fauteurs de troubles intestins sont les «excréments » du royaume.

Si le souverain est une "tirelire vitale ", c'est qu'il détient le monopole des substances des ancêtres, capital symbolique du royaume. Les royautés sacrées des Grassfields sont en effet le produit d'un processus d'accumulation économique et de centralisation politique dont Jean-Pierre Warnier et Nicolas Argenti retracent l'histoire sur la longue durée. Leur émergence serait bien antérieure à la traite atlantique: les hautes terres sont en effet un important centre de civilisation bien avant leur intégration comme périphérie d'un système-monde centré sur l'Europe atlantique (Warnier 1985). Les sociétés des Grassfields se sont progressivement structurées en cités-États au sein d'un vaste espace régional caractérisé par un intense brassage de populations. Ce processus suppose la délimitation de clôtures qui confere une certaine forme d'intériorité et d'autonomie à des entités politiques en y territorialisant des personnes et des biens autrement mobiles. On comprend ainsi pourquoi les systèmes politiques des Grassfields accordent une telle importance aux enveloppes et à leurs limites. C'est alors la métallurgie, présente depuis plus de deux millénaires dans la région, qui aurait permis l'accumulation de richesses nécessaire à l'émergence de royaumes. L'importance de la métallurgie comme fondement matériel et symbolique des royautés sacrées est d'ailleurs attestée plus au sud en Afrique centrale (Vansina 1990).

La traite atlantique a sans aucun doute favorisé la centralisation politique des royaumes des Grassfields. L'insertion des cités-États dans une économie globale fondée sur des réseaux de commerce de longue distance accentue la stratification sociale au point d'aboutir à une véritable hypertrophie des hiérarchies de palais. Ce n'est pas un hasard si le trésor royal contient des biens de prestige, le plus souvent d'origine européenne, qui proviennent du commerce de longue distance. Le commerce d'esclaves occupe alors une place centrale dans l'économie politique des Grassfields. Les mythes d'origine racontent comment l'ancêtre fondateur, fuyant des négriers à cheval, s'est installé dans une nouvelle région pour y fonder le royaume. À l'exception des razzias peules, bamum ou chamba, la fourniture d'esclaves ne se fait pourtant pas par des raids armés menés par des 
étrangers, mais plutôt par la vente de parents ou de voisins. À partir du XVIII ${ }^{\mathrm{e}}$ siècle, les notables des palais se mettent en effet à vendre en esclavage les cadets de leur propre lignage, afin d'acquérir en échange des biens de prestige. Il s'agit ainsi d'un système de «traite sans raids » (Warnier 1989). Cette insidieuse prédation interne est pensée comme une forme d'endocannibalisme: les aînés "mangent" les cadets. Bien que la colonisation allemande (à partir de 1884) se fasse au nom de la lutte contre l'esclavage, celui-ci ne décroît que très lentement dans la région. Le travail forcé imposé par les colons qui ont besoin de main-d'œuvre pour les plantations ou le portage caravanier s'inscrit de toute façon dans la continuité de la période précoloniale. Les élites des palais tirent profit du système du travail forcé comme auparavant de la traite d'esclaves: elles livrent aux colons des cohortes de cadets en échange du maintien de leur autorité coutumière. Les mandats français et anglais après la Première Guerre mondiale ne mettent pas fin à cette situation. Et la période postcoloniale qui s'ouvre après 1960 perpétue encore cette domination : les notables des palais, reconvertis dans le parti unique, investissent l'appareil d'État afin d'en capter les ressources au détriment des cadets (le roi mankon occupe par exemple des fonctions politiques à l'échelle nationale). La gouvernementalité du roi-pot se prolonge ainsi à l'échelle nationale dans une "politique du ventre» (Bayart 1990) qui joue sensiblement sur le même imaginaire du corps. Comme le souligne bien Nicolas Argenti, l'État colonial et postcolonial a en définitive largement reproduit les inégalités des gérontocraties locales.

Cette alliance entre les autorités traditionnelles et l'État participe d'une réinvention de la tradition monarchique au service d'une modernité politiquement conservatrice. Ce "retour des rois» (Perrot \& Fauvelle-Aymar 2003) pose la question de l'insertion des chefferies des Grassfields dans l'État camerounais. Comblant une lacune de l'édition anglaise (The PotKing, 2007), Jean-Pierre Warnier consacre le chapitre conclusif de l'édition française à la situation contemporaine de la royauté mankon. Dans un contexte politique où le royaume n'existe plus que comme un sous-ensemble subordonné de l'État-nation, la royauté peut-elle être autre chose qu'une institution en voie de folklorisation? Le roi a toutefois su jouer habilement des principes de l'analogisme fractal pour adapter la royauté à ce changement d'échelle. Si la tranchée qui délimitait autrefois les frontières de la cité a perdu sa raison d'être, c'est désormais le site web de l'association MACUDA (Mankon Cultural Development Association), dont le roi est le président fondateur, qui représente une enveloppe virtuelle susceptible de rassembler les élites mondialisées du royaume, désormais expatriées en Europe ou aux États-Unis. L'inauguration du 
musée mankon en 2005 résume bien le processus de patrimonialisation culturelle qui accompagne ces changements. Cette patrimonialisation muséale, qui participe d'une politique d'autochtonie définie à l'échelle de l'État camerounais avec l'appui d'ONG internationales, témoigne des glissements qu'opère la redéfinition contemporaine de la royauté et des tensions possibles que cela peut susciter. Les regalia exposés dans le musée qui jouxte le palais sont le patrimoine indivis du royaume : le roi en est le gérant pour le bien de tous. Fondé sur un régime juridique de la propriété distinct du droit coutumier, l'acte de création du musée stipule pourtant que le souverain est le propriétaire du trésor royal en son nom propre, au risque d'exposer sa légitimité à la contestation ouverte de ses sujets.

\section{Les cadets, de l'adhésion à la révolte}

Les royautés des Grassfields reposent donc historiquement sur l'exploitation et la domination des cadets, condition même de leur existence. Cela passe entre autres par la grande polygamie de l'aristocratie des palais : certains rois ont pu avoir plusieurs centaines d'épouses. Cela implique qu'une part importante de la population masculine ne peut se marier et même, étant donné la rigueur des interdits sur les relations hors mariage, était jusqu’à peu privée de toute sexualité selon Jean-Pierre Warnier ${ }^{2}$. Il estime ainsi qu'entre un tiers (à l'époque de la traite) et la moitié (au début de l'ère coloniale) de la population masculine était condamnée au célibat à vie. Comment la royauté a-t-elle alors pu se maintenir face à une telle inégalité de condition? La frustration des cadets célibataires ne devraitelle pas entraîner nécessairement leur rébellion contre le palais? JeanPierre Warnier soutient qu'il n'en est rien : la royauté mankon susciterait au contraire une adhésion générale, y compris des cadets. La domination du palais pousse à son comble l'idéologie de la séniorité : tous les subordonnés du palais sont d'éternels cadets assujettis à leurs aînés. L'aînesse est affaire de statut. En l'absence de toute initiation masculine, c'est le mariage qui fait l'homme et non l'inverse. Aussi âgés soient-ils, les cadets célibataires ne sont donc que des "enfants " et se percevraient eux-mêmes comme tels: ils auraient en effet une image inconsciente de leur propre corps qui serait complètement asexuée. Le célibat ne serait donc pas vécu par les cadets comme une privation. Pour étayer empiriquement ces conjectures, il manque cependant une ethnographie précise de la sexualité, de ses pratiques ordinaires et des techniques par lesquelles passe son

2. Assimiler célibat et chasteté forcée suppose toutefois un arrimage de la sexualité au mariage qui est loin d'aller de soi : interdire l'adultère ne le rend pas impossible, cela le rend clandestin. On ne peut donc faire l'impasse sur la question de l'écart entre les normes morales et les pratiques réelles. 
contrôle par le palais. Mais Jean-Pierre Warnier reconnaît qu'il a dû, malgré lui, arrêter son enquête de terrain au seuil des chambres à coucher. Si l'hypothèse des cadets asexués paraît tout de même quelque peu improbable, on peut cependant imaginer un scénario alternatif qui rende compte du contrôle de la sexualité par les aînés : les hommes célibataires ont accès, clandestinement, à la sexualité ; mais les élites polygames s'attribuent leur progéniture, les privant ainsi de descendance. Par ce biais, les aînés confisquent le potentiel reproducteur des cadets à leur seul profit.

Jean-Pierre Warnier souligne que la valeur symbolique associée aux actions portant sur les substances vitales contribue également à masquer la domination en la travestissant. Forme typique de la fausse conscience, ce sont en réalité les cadets qui se perçoivent en dette vis-à-vis de leur souverain, car ils reçoivent de sa part ces biens inestimables que sont les substances des ancêtres en échange de biens plus ordinaires (du labeur, du bétail, des femmes). Ils se satisfont donc de cet échange qui n’est pas perçu comme une transaction de dupes. Le roi est d'ailleurs lui-même assujetti au système dont il est le sommet, tel Louis XIV au sein de la société de cour (Elias 1985) : alimenter ses sujets en substances vitales et s'occuper de ses nombreuses femmes est une lourde charge, dans laquelle n'intervient aucune notion de plaisir, ni d'intérêt personnel. La domination du palais fait ainsi appel au consentement plus qu'à la contrainte - et c'est en cela qu'elle s'exerce sur un mode hégémonique. Elle est intériorisée par les cadets au point d'échapper en bonne partie à la prise de conscience critique et d'être acceptée sans frustration.

L'argumentation de Jean-Pierre Warnier repose toutefois sur une analogie, sans doute un peu trop rapide pour être pleinement convaincante, entre savoir procédural associé à des conduites motrices et savoir implicite associé aux relations de pouvoir. Faire du vélo nécessite assurément un savoir procédural mis en œuvre de manière automatique et inconsciente sous forme de schèmes moteurs incorporés. Si ce n'était pas le cas, le cycliste tomberait. Mais peut-on vraiment affirmer que l'exercice des relations de pouvoir passe par un savoir procédural du même ordre que la maîtrise technique? C’est bien la "valeur " prêtée à la salive du souverain qui confere son efficacité à la pulvérisation royale et, partant, rend possible l'adhésion des cadets. Jean-Pierre Warnier note d'ailleurs que les techniques de pouvoir s'accompagnent souvent de paroles dotées d'une valeur performative; ces performances verbales restent toutefois trop peu explorées dans l'ouvrage qui n'accorde sans doute pas toute la place qu'elle mériterait à l'étude de détail de l'enchâssement du langage dans l'action. L'exercice des relations de pouvoir ne relève donc pas d'une efficacité technique à strictement parler, même si elle peut être perçue comme telle 
par les acteurs eux-mêmes (d'où la nécessité de maintenir la distinction entre les descriptions emic et etic). C'est pourquoi des expressions comme «technologie de pouvoir» ou "physiologie du pouvoir» sont au moins partiellement trompeuses si on les prend trop au pied de la lettre. Si on ne peut qu'être en sympathie avec la charge menée par Jean-Pierre Warnier contre une approche réduisant le corps et la matérialité à un système de signes abstraits, on ne saurait cependant dissoudre totalement le sémiologique dans l'acide praxéologique. Il conviendrait par conséquent de poursuivre plus avant l'analyse des ressorts de l'efficacité des actions symboliques associées aux relations de pouvoir et des processus cognitifs que ces actions mobilisent. Il est par exemple manifeste que la gouvernementalité du roipot repose sur une mobilisation massive du raisonnement analogique. Que le corps constitue bien le noyau matriciel de ce raisonnement analogique ne signifie pas pour autant que les schèmes cognitifs inconscients qui gouvernent ce raisonnement soient strictement du même ordre que des schèmes moteurs automatiques. L'ambiguïté de l'argumentation de Jean-Pierre Warnier provient sans doute d'un certain flottement dans l'usage de la notion d' "inconscient ", écartelé entre psychanalyse, psychologie cognitive et physiologie motrice. Si la gouvernementalité du roi-pot repose sur un "inconscient moteur et cognitif ", tout le problème repose justement sur l'articulation de ce «et». Il faudrait ainsi mieux distinguer entre les techniques du corps stricto sensu et les techniques de pouvoir, entre ce qui passe par des routines motrices automatiques et ce qui nécessite un traitement cognitif à un niveau supérieur. De même, il conviendrait de mieux distinguer entre ce qui est totalement inconscient et ce qui est seulement tacite ou implicite : ce qui va habituellement sans dire peut en effet, dans certaines conditions, faire l'objet d'une prise de conscience. L'anthropologie politique que nous propose Jean-Pierre Warnier ne saurait ainsi se dispenser d'une exploration précise des passages de l'implicite à l'explicite dans la cognition et des différents paliers entre savoir procédural et savoir déclaratif (Karmiloff-Smith 1992).

Ces précisions permettraient sans doute d'affiner, voire de nuancer, la thèse de l'intériorisation inconsciente de la domination et de l'impossibilité de toute prise de conscience critique. Jean-Pierre Warnier mentionne d'ailleurs lui-même au détour d'un paragraphe la possibilité, certes marginale, de trajectoires individuelles permettant une forme de réflexivité critique. Il fait allusion à des artistes - ainsi ce mystérieux "roi-sculpteur de Kom»- et à des devins-guérisseurs, mais aussi à des "penseurs" et à des " "mères" au tempérament bien trempé, dont la truculence, l'ironie et les réparties emportaient le respect de leur entourage" (p. 282). On aurait cependant aimé en savoir plus sur ces 
individualités singulières et sur les conditions de leur émergence. Il semble bien que ce soit leur position décalée qui permette à ces individus le recul nécessaire à une prise de conscience : ainsi le devin-guérisseur qui se situe hors lignage, le sculpteur qui parvient à se distancier à travers ses œuvres, ou encore le jeu corrosif du rire. On peut en outre se demander si la lucidité singulière que Jean-Pierre Warnier prête à ces individus qu'il nomme précisément et qu'il connaît donc bien ne tiendrait pas en réalité à la relation privilégiée qu'il a eue avec eux sur le terrain et aux confidences intimes qu'il a ainsi pu recueillir. L'absence habituelle de verbalisation concernant la domination du palais tiendrait alors moins à son impossible appréhension consciente qu'au fait que certaines affaires sensibles ne peuvent être abordées avec n'importe qui et n'importe comment: elles doivent rester tues, alors même qu'elles sont très largement sues (Zempléni 1984, 1996). Une anthropologie des rapports de pouvoir ne peut donc se passer d'une analyse réflexive attentive aux situations d'interlocution et, en creux, au rôle du silence tacite.

Préciser les passages possibles de l'implicite à l'explicite permettrait également de mieux rendre compte du changement. Comment une forme de domination échappant à l'appréhension consciente pourrait-elle en effet être remise en cause? Jean-Pierre Warnier décrit pourtant une rupture brutale dans les années 1980-1990 : refusant de rester enfermés dans leur condition de cadets asexués, la jeunesse s'émancipe ouvertement de la gouvernementalité sexuelle du roi-pot. Cette révolution sexuelle qui menace les principes mêmes de la royauté coïncide avec l'agitation politique menée par le Social Democratic Front, parti d'opposition au Président Biya au pouvoir depuis 1982 : les partisans du SDF sont en effet des jeunes hommes pour la plupart. Les Grassfields anglophones, fief du SDF, font alors l'objet d'une violente répression par l'État, durcissant encore un peu plus les tensions entre les cadets et les élites. Les années 1990 marquent ainsi un décrochage des cadets par rapport à la royauté qu'ils semblaient jusque-là soutenir très largement: en 2000, le roi mankon doit installer des grilles à son palais pour contenir les violences. Mais les cadets ont-ils vraiment attendu la fin du XXe siècle pour prendre conscience de leur oppression et se révolter? Jean-Pierre Warnier soutient cette hypothèse qui l'amène d'ailleurs à réinterpréter ses précédents travaux (1996) sur les révoltes dans les Grassfields à la lumière des recherches de Dominique Malaquais (2002) pour montrer que ces mouvements ne traduisaient en réalité pas une rébellion des cadets contre la royauté. Nicolas Argenti donne pourtant une autre interprétation de ces mêmes événements et insiste sur l'importance des révoltes des cadets dans l'histoire des Grassfields. 
À l'époque précoloniale, la menace de la vente en esclavage ou du poison d'épreuve a sans aucun doute contribué à décourager les velléités de rébellion (la coercition n'est donc pas absente des techniques de pouvoir, même si Jean-Pierre Warnier a tendance à la minorer). La défection représentait en outre une soupape de sécurité. La démographie des Grassfields se caractérise historiquement par une importante population flottante de cadets qui espèrent échapper à leur célibat en migrant d'une chefferie à l'autre. Ils sont alors intégrés à des lignages locaux comme les "enfants" classificatoires des nouveaux maîtres pour lesquels ils travaillent, espérant obtenir en échange la possibilité de se marier un jour. À l'époque coloniale, la défection des cadets devient une stratégie plus intéressante: les jeunes hommes abandonnent les chefferies pour rejoindre les écoles missionnaires ou chercher du travail salarié en ville, dans les plantations ou dans les mines. Ce départ représente une possibilité d'émancipation de la tutelle des aînés. Partis travailler à l'extérieur du royaume, les free boys refusent ainsi de reverser leur salaire au roi, comme ils devraient normalement le faire, entrant ainsi en rébellion ouverte contre le pouvoir. Au tournant du XXe siècle apparaissent les premières révoltes violentes des cadets contre les chefferies avec le mouvement des tapenta. Il s'agit d'une troupe de soldats irréguliers, devenus mercenaires à leur propre compte après avoir rompu les attaches avec leur chefferie d'origine et les autorités coloniales. Portant uniformes et fusils allemands, ils mettent la région à feu et à sang. Le terme "tapenta " provient de l'anglais interpreter et désignait à l'origine les traducteurs au service de l'administration coloniale; il a ensuite servi à qualifier toute personne parlant une langue européenne ou, plus largement, maitrisant le savoir des Blancs sous quelque forme que ce soit. C'est ainsi en s'appropriant les ressources des Blancs - leur langue, leurs habits et leurs fusils - que les cadets en rupture de ban trouvent les moyens de s'affranchir des chefferies. Les révoltes des cadets se répètent tout au long $\mathrm{du} \mathrm{XX}$ siècle dans les Grassfields. Ainsi la rébellion des «maquisards » de l'Union des populations du Cameroun des années 1950-1960 (Mbembe 1996) : il s’agit à la fois d'une insurrection anti-coloniale et d'une révolte des cadets contre les élites des chefferies, alliées du pouvoir colonial. En insistant sur l'importance des révoltes des cadets, Nicolas Argenti retrace ainsi l'émergence d'une nouvelle force politique: la «jeunesse». Il s'agit d'une catégorie liminale, née de l'incapacité de l'État tant colonial que postcolonial à offrir une alternative à la séniorité traditionnelle, et rassemblant ceux qui refusent de rester des "enfants " assujettis aux élites, mais qui ne peuvent pour autant accéder au statut d'"aînés" et se retrouvent ainsi entravés dans leurs aspirations d'émancipation. 
Jean-Pierre Warnier et Nicolas Argenti nous livrent en définitive deux versions très différentes de la situation des cadets. Certes, chaque micro royaume des Grassfields est singulier. Oku, plus isolé dans la montagne, offre moins de possibilités d'échappatoire: les tensions entre aînés et cadets y sont donc plus vives. À Mankon, les querelles intestines se sont davantage focalisées sur un conflit de succession au sein même de l'aristocratie du palais (entre 1920 et 1960). Cependant, la divergence entre les deux auteurs est avant tout une question de perspective. Jean-Pierre Warnier adopte d'une certaine façon le point de vue du roi $^{3}$ : il décrit les cadets comme des récipients vides qui dépendent du pouvoir dispensateur $\mathrm{du}$ souverain pour exister. Les notables du palais sont ainsi les seuls à posséder une véritable capacité d'action. Nicolas Argenti s'attache à restituer la perspective des cadets de manière plus charitable. Refusant d'en faire de simples récipients passifs, il leur accorde une plus grande capacité d'action politique. Il s'inscrit en ce sens dans le récent courant de recherches consacrées aux enfants et aux adolescents dans l'Afrique postcoloniale, qui insiste sur le fait que ces derniers représentent une force active qui occupe désormais une place centrale dans l'espace public (Honwana \& de Boeck 2005). Alors que Jean-Pierre Warnier donne une version finalement très bourdieusienne de la domination et de son intériorisation par les dominés, Nicolas Argenti en donne une version plus foucaldienne qui laisse la place à l'analyse des pratiques de résistance qui se logent dans les interstices des rapports de pouvoir. Les cadets ne sont pas des corps disciplinés au point de ne pouvoir résister. Si une domination hégémonique pénètre les corps et les esprits au point d'échapper en bonne partie à la conscience critique, les pratiques de résistance qu'elle suscite peuvent toutefois se situer elles aussi dans cette même zone grise de la conscience vague, "espace entre le conscient et l'inconscient" (Comaroff \& Comaroff 1991 : 29). En effet, la rébellion ne passe pas nécessairement par un discours critique clairement articulé, mais peut également s'exprimer dans des pratiques non discursives plus ambiguës - ce que James C. Scott (2009) appelle le domaine de «l'infra-politique». Contre les théories de l'hégémonie qui dominent la sociologie critique, cet auteur souligne l'importance des pratiques souterraines de résistance qui se cachent derrière les discours (ou les silences) publics des groupes subalternes. Attentif à ces " armes des faibles " (pour citer le titre d'un précédent ouvrage de James Scott paru en 1985), Nicolas Argenti reprend ainsi

3. Il est par exemple flagrant qu'il adopte le point de vue des élites polygames sur la sexualité des cadets, ou plutôt sur leur absence supposée de sexualité. Lorsque les cadets accèdent de manière ouverte à la sexualité dans les années 1980-1990, les aînés se lamentent alors de la «dépravation morale» des jeunes générations à laquelle ils attribuent l'épidémie de VIH/SiDA. 
la question de l'adhésion ou de la résistance des cadets posée par JeanPierre Warnier, mais propose de décaler le regard en s'intéressant aux danses de masques. Les mascarades des Grassfields sont en effet le lieu d'une tension persistante entre les aînés et les cadets.

\section{Mascarades ambiguës}

Se démarquant de l'approche fonctionnaliste du rituel comme instrument au service de l'ordre social, Nicolas Argenti insiste sur l'imagination historique que les mascarades expriment. Il s'inscrit ainsi dans la perspective des Comaroff sur le «rituel comme pratique historique» (Comaroff 1985: 194) et s'inspire plus particulièrement de l'ouvrage de Rosalind Shaw, Memories of the Slave Trade (2002), qui montre comment la mémoire de la traite négrière au Sierra Leone passe moins par des discours explicites que par l'imaginaire de la possession par les esprits, de la divination et de la sorcellerie. De même dans les Grassfields, la violence fondatrice des royaumes, la terreur de l'esclavage et de la colonisation, la brutalité de l'antagonisme entre les cadets et les élites ne font aujourd'hui l'objet d'aucun discours explicite. Ce sont en fait les danses de masques qui témoignent à leur manière de ce passé violent mais silencieux. En effet, la mémoire sociale des événements passés - surtout lorsqu'ils sont traumatisants - ne relève pas nécessairement de la mémoire déclarative, mais peut tout aussi bien mobiliser la mémoire procédurale. Les mascarades offrent ainsi, à qui sait les lire, une « récapitulation de l'histoire des Grassfields»(p. 242). En envisageant les danses de masques comme la mémoire incarnée du passé des Grassfields, Nicolas Argenti rejoint par conséquent l'approche de Jean-Pierre Warnier, centrée sur les pratiques non discursives et la culture incorporée. À vouloir déchiffrer les significations implicites des mascarades, il s'expose cependant du même tenant au risque de la surinterprétation (Olivier de Sardan 1993) ${ }^{4}$.

Les danses de masques sont omniprésentes dans les Grassfields. Les plus importantes d'entre elles sont les mascarades officielles qui ont lieu pendant les festivals royaux. De nombreux masques s'y donnent en spectacle : Nkok qui représente une bête sauvage, Mabu le bourreau du palais, etc. Le terme qui signifie "masque" (kekúm) possède un champ sémantique qui dépasse l'objet lui-même, car il désigne également des personnages non masqués, comme par exemple les bouffons Nokan. Les porteurs

4. Les adeptes du culte Haouka, né dans les années 1920 au Niger, sont possédés par des esprits liés au monde colonial. Paul Stoller (1995), à la suite de Jean Rouch (1955), y voit une «comédie horrifique" qui moque avec un humour cruel le pouvoir blanc. La résistance au colonisateur s'exprimerait ainsi directement dans le spectacle violent des corps possédés - interprétation politique que Jean-Pierre Olivier de Sardan critique ou, du moins, nuance. 
de masque sont des notables du palais qui payent parfois fort cher pour intégrer une société secrète de masques. Évocation implicite du passé, les mascarades remémorent notamment la traite des esclaves. Selon Nicolas Argenti, les cauris qui ornent les costumes représentent la monnaie qui servait d'unité de valeur pendant la période de la traite. De même, les épées portées par des masques effrayants qui chargent l'assistance rappellent les razzias des négriers peuls. Les masques incarnent en réalité des entités ambiguës : Mabu est à la fois une proie et un prédateur, un autochtone et un étranger, un esclave et un négrier. Cette ambivalence passe par tout un jeu de condensations et d'inversions rituelles qui sont d'autant plus ambiguës qu'elles restent implicites - procédé que Nicolas Argenti appelle "l'indétermination poétique » des mascarades. La formule n'est sans doute pas très heureuse, dans la mesure où l'ambivalence des mascarades tient moins à un prétendu flou poétique qu'à une dynamique interactionnelle aussi précise qu'efficace, comme le montre bien le comportement des masques à l'égard de l'assistance. Ainsi, les acolytes qui escortent le masque Aga sollicitent des offrandes avec une insistance muette : une main tendue, l'autre brandissant une lance, ils sourient énigmatiquement, les yeux écarquillés. Cette posture paradoxale condense une série d'attitudes antithétiques : la lance contredit la main tendue et le sourire; le caractère impérieux de la sollicitation contredit le fait qu'il s'agisse d'une offrande. Les auxiliaires du masque se placent ainsi dans une posture qui relève simultanément de la soumission et de la domination vis-à-vis des spectateurs qu'ils abordent ${ }^{5}$. Ce comportement déstabilisant (de la part de notables qui plus est) illustre bien la manière singulière dont les mascarades donnent à voir la domination du palais.

Figures liminales associées à la fois à l'univers de la cité et à l'univers sauvage de la forêt, les masques sont en effet à l'image du roi lui-même. Les mythes attribuent une origine étrangère à celui qui est pourtant le fondateur de l'autochtonie du royaume. Le souverain, protecteur et nourricier, est également un dangereux prédateur: la panthère est d'ailleurs son double animal. Les mascarades ne sont donc pas une représentation transparente de l'histoire des Grassfields, mais reposent sur une série de déplacements implicites. Elles représentent le pouvoir du palais comme s'il était une défense nécessaire contre la menace d'agresseurs extérieurs (razzias de négriers ou sorciers cannibales associés au monde de la forêt). Les masques représentent à la fois la menace dont le palais protège les sujets et la violence au moyen de laquelle il les protège. Mais cela oblitère du même tenant le fait que ce sont en réalité les élites du palais qui

5. Sur la condensation paradoxale dans le rituel, cf. Houseman \& Severi (1994). 
exercent une violence intestine à l'égard des sujets de leur propre royaume : en les réduisant en esclavage pour s'enrichir ou en les accusant de sorcellerie pour faire taire les indociles. Le palais se présente ainsi comme le protecteur de ceux-là même qu'il terrorise. Les mascarades officielles participent par conséquent de la légitimation du pouvoir du palais : elles mettent en scène la violence du passé en la travestissant, en la "masquant " pourrait-on dire.

Du fait même de leur ambivalence, les danses de masques peuvent néanmoins faire l'objet d'une double lecture. Nicolas Argenti s'intéresse ainsi à la façon dont les cadets eux-mêmes perçoivent les cérémonies officielles, à rebours de la version hégémonique du palais. Les mascarades offrent en effet un registre de choix pour examiner le rapport ambigu entre la soumission et l'insoumission des cadets. Selon Nicolas Argenti, si les cadets accourent par milliers aux festivals royaux, c'est que le spectacle des masques terrifiants chargeant l'assistance mettrait crûment en lumière la violence que leur fait subir le palais : loin de masquer leur oppression, il la démasquerait au contraire. De leur point de vue, les mascarades ne sont donc pas une innocente commémoration folklorique, mais un spectacle d'une brûlante actualité : elles manifestent un passé de violence et d'oppression qui se continue dans le présent. À travers les mascarades, les cadets peuvent revivre la répétition du passé, non pas sur le mode du traumatisme, mais sur un mode plus ambigu qui associe la terreur et le plaisir. En somme, les cadets parviendraient à jouir du spectacle de leur domination. La démonstration de Nicolas Argenti, parfois trop psychologisante, n'emporte toutefois pas entièrement la conviction. Quoiqu'il s'en défendrait très certainement, son argumentation suppose une théorie de la "sublimation" qui n'est pas véritablement étayée, alors même qu'il entend se démarquer du modèle cathartique des «rites de rébellion » selon Max Gluckman $(1963)^{6}$ : les cadets parviendraient à trouver du plaisir dans une représentation de la terreur, dans la mesure où celle-ci ne passe pas par un discours explicite, mais par une performance esthétique qui joue sur l'implicite et l'ambiguïté. En réalité, la séduction qu'exercent les mascarades du palais sur les cadets est moins une affaire de catharsis que, là encore, de dynamique interactionnelle. Les mascarades reposent sur l'articulation des conduites manifestes des notables masqués et des cadets les uns envers les autres: l'implication de chacune des deux parties s'appuie sur la perception du comportement de l'autre partie à son propre égard ${ }^{7}$. Même s'ils ne

6. Max Gluckman voit dans les « rites de rébellion » une soupape de sûreté qui permet aux tensions sociales de s'exprimer dans un cadre contrôlé.

7. Sur la dynamique relationnelle des mascarades, cf. Bonhomme (2006: 182-184). Voir aussi Smith (1984); Houseman (2002). 
sont que spectateurs, les cadets jouent un rôle crucial : sans eux, les mascarades perdent leur sens. Pour manifester leur pouvoir, les notables du palais ont besoin des cadets qui fuient devant leurs masques. Mais les cadets ne sont pas entièrement dupes du rôle qu'on leur fait jouer. Ils peuvent même s'imaginer que ce sont en fait eux qui dupent le palais en jouant son jeu. C'est cet écart entre les perspectives des deux parties qui rend possible le plaisir ambigu que les cadets peuvent tirer de la participation au spectacle de leur sujétion par les masques du palais. Les attitudes émotionnelles et épistémiques des cadets envers les mascarades sont, en définitive, les produits émergents d'une dynamique interactionnelle sous-jacente.

Les grandes mascarades du palais ne représentent cependant qu'une fraction des innombrables danses de masques des Grassfields. Chaque chefferie, chaque lignage, chaque village possède également ses propres masques. Ces mascarades villageoises sont subordonnées à celles du palais, qui leur servent de modèle: tout nouveau masque doit en effet être approuvé par le roi. Cela n'en fait pas pour autant de simples décalques des danses de masques du palais. Même si elles sont sous le contrôle des aînés, les mascarades villageoises font l'objet d'une appropriation par les cadets : ce sont eux qui en sont les membres les plus actifs et qui inventent constamment de nouveaux masques. Les cadets ne sont donc plus cantonnés dans le rôle de spectateurs, mais sont des acteurs à part entière des mascarades. Au village, les masques apparaissent notamment lors des retraits de deuil. Comme souvent en Afrique subsaharienne, ces secondes funérailles sont vécues sur le mode des réjouissances: il s'agit en effet de "danser la mort» (alors que lors des funérailles, on "pleure la mort»). Les mascarades villageoises se révèlent ainsi nettement plus festives que celles du palais, plus violentes et plus sinistres. Alors que les cérémonies du palais évoquent l'exclusion des cadets du mariage, les spectacles villageois sont l'occasion de jeux de séduction entre garçons et filles. Nicolas Argenti montre en outre comment les mascarades villageoises sont mises au service de rites féminins de fécondité, s'attachant ainsi à restituer le point de vue des femmes, davantage absentes du livre de Jean-Pierre Warnier (qui a tendance à rabattre la perspective des femmes sur celle, dominante, des hommes).

D'autres danses, d'origine récente, expriment plus nettement encore l'autonomie des dominés vis-à-vis du palais (Argenti 1998, 2001, 2004). Il s'agit de mascarades organisées par des femmes ou des jeunes hommes, souvent interdites par le palais et portant des noms évocateurs (tel le groupe "Mondial»). Dans les Grassfields des années 1960-1970, un groupe de danse nommé "Air Youth» remplace ainsi les masques traditionnels par un accoutrement militaire et une esthétique moderne. 
En mimant les « gendarmes » de sinistre réputation qui incarnent la violence de l'État postcolonial, cette nouvelle danse représente une tentative d'appropriation imaginaire du pouvoir de l'État par les cadets opprimés.

Les jeunes enfants jouent également à faire des mascarades comme celles des adultes. Entièrement organisées par des enfants (entre trois et douze ans), ces danses échappent alors totalement au contrôle des aînés. Ces mascarades enfantines mettent en scène des militaires, le parti d'opposition du SDF ou encore des Blancs, sur une chorégraphie inspirée de la musique populaire. Elles permettent aux enfants de s'approprier sur un mode ludique le monde dans lequel ils vivent, aussi bien la violence politique que la modernité marchande. Il est d'ailleurs révélateur que, contrairement aux adultes, les enfants ne fabriquent pas leurs masques avec des éléments provenant du monde de la forêt, mais avec des « détritus de la modernité » tels que des vieux bidons ou des cartons.

On pourrait penser que ces imitations des mascarades officielles témoignent de l'aliénation des dominés incapables de sortir du modèle hégémonique imposé par le palais. Ce sont en réalité des réappropriations originales qui ne laissent pas indemne le modèle officiel (sur ces questions, cf. Bonhomme 2010). En faisant référence à Mikhail Bakhtine (1970), auteur incontournable d'une certaine anthropologie postmoderne, Nicolas Argenti souligne ainsi que les cadets, les femmes et les enfants n'imitent les mascarades du palais que pour mieux les détourner de manière carnavalesque: il s'agit en quelque sorte de mascarades de mascarades, parodies festives qui offrent aux dominés un espace de contestation possible. Cette transformation mimétique possède un effet cathartique : le rire carnavalesque crée un décalage réflexif qui permet de mettre à distance la domination du palais - ce que Jean-Pierre Warnier notait lui aussi au passage à propos des éclats de rire de ces femmes "au tempérament bien trempé». Dans l'Afrique postcoloniale, la dérision grotesque sert en effet souvent d'arme des faibles: les dominés se moquent du pouvoir en le mimant à l'excès (Mbembe 1992 ; Toulabor 1981). En définitive, Nicolas Argenti lit dans les mascarades villageoises un "contre-discours" des cadets face au discours hégémonique du palais. Reste alors en suspens la question du rapport ambigu entre ces danses de masques et les mouvements de révolte plus frontaux: les mascarades relèvent-elles d'une résistance "réelle", "symbolique» ou "imaginaire" (sur ces questions, cf. Althabe 1969)? Quelle est en somme la portée politique du " contre-discours " véhiculé par les masques des cadets? On peut d'ailleurs noter que ce terme n'est sans doute pas le plus approprié pour qualifier des performances rituelles qui ne passent justement pas par un discours articulé. Tout se passe comme si la métaphore linguistique, chassée par la porte, revenait par la fenêtre. 
Ce travers se retrouve d'ailleurs dans l'ouvrage de Jean-Pierre Warnier lorsqu'il lit dans les corps des sujets la "Constitution écrite» du royaume mankon (p. 280). Ces lapsus révélateurs témoignent bien de la difficulté qu'une anthropologie habituée à "entextualiser » la culture éprouve à rendre compte des pratiques non discursives. Il n'en reste pas moins que les ouvrages de Jean-Pierre Warnier et de Nicolas Argenti constituent, chacun à leur manière, des contributions importantes et stimulantes pour saisir comment, à travers des pulvérisations de vin de palme ou des danses de masques, le pouvoir pénètre les corps aussi bien que les esprits, mais également pour appréhender les modalités ambiguës de la participation ou de la résistance des dominés à la domination.

Musée du quai Branly Département de la recherche et de l'enseignement, Paris Julien.Bonhomme@quaibranly.fr

MOTS CLÉS/KEYWORDS : pouvoir/power - corps/body - domination - roi/king - royauté/kingship - cadet/youngest boy - mascarade/masquerade - Grassfields - Cameroun/Cameroon. 
Adler, Alfred

1982 La Mort est le masque du roi.

La royauté sacrée des Moundang du Tchad.

Paris, Payot.

Althabe, Gérard

1969 Oppression et libération dans

l'imaginaire. Paris, Maspero.

\section{Anzieu, Didier}

1985 Le Moi-Peau. Paris, Dunod.

\section{Argenti, Nicolas}

1998 "Air Youth : Performance, Violence and the State in Cameroon ", Journal of the Royal Anthropological Institute 4 (4): 753-782.

2001 "Kesum-Body and the Places of the Gods : The Politics of Children's Masking and Second-World Realities in Oku (Cameroon) ", Journal of the Royal Anthropological Institute 7 (1) : 67-94.

2004 «La danse aux frontières:

les mascarades interdites des femmes et des jeunes à $\mathrm{Oku}$ ", in Jean-François Bayart \& Jean-Pierre Warnier, eds, Matière à politique. Le pouvoir, les corps et les choses. Paris, Karthala : 151-179.

Bakhtine, Mikhail

1970 L'Euvre de François Rabelais et la culture populaire au Moyen Âge et sous la Renaissance. Paris, Gallimard.

\section{Bayart, Jean-François}

1990 L'État en Afrique: la politique du ventre. Paris, Fayard.

\section{Bayart, Jean-François}

\& Jean-Pierre Warnier, eds

2004 Matière à politique. Le pouvoir, les corps et les choses. Paris, Karthala.

\section{Berthoz, Alain}

1997 Le Sens du mouvement.

Paris, Odile Jacob.
Bloch, Maurice

1998 How We Think They Think.

Anthropological Approaches to Cognition, Memory, and Literacy. Boulder,

Westview Press.

Bonhomme, Julien

2006 Le Miroir et le Crâne. Parcours initiatique du Bwete Misoko (Gabon). Paris, CNRS Éd. - Éd de la MsH.

2010 "Masque Chirac et danse de Gaulle : images rituelles du Blanc au Gabon », Gradhiva 11. [À paraître.]

\section{Bourdieu, Pierre}

1972 Esquisse d'une théorie de la pratique.

Genève, Droz.

\section{Comaroff, Jean}

1985 Body of Power, Spirit of Resistance. The Culture and History of a South African People. Chicago, University of Chicago Press.

\section{Comaroff, John L. \& Jean Comaroff}

1991 Of Revelation and Revolution.

Christianity, Colonialism, and Consciousness in South Africa. Chicago, University of

Chicago Press.

Csordas, Thomas J., ed.

1994 Embodiment and Experience.

The Existential Ground of Culture and Self.

Cambridge, Cambridge University Press.

De Heusch, Luc

1972 Mythes et rites bantous. Le Roi ivre ou l'Origine de l'État. Paris, Gallimard.

1982 Mythes et rites bantous. Rois nés d'un cour de vache. Paris, Gallimard.

2000 Mythes et rites bantous.

Le roi de Kongo et les monstres sacrés.

Paris, Gallimard.

Descola, Philippe

2005 Par-delà nature et culture.

Paris, Gallimard. 
Elias, Norbert
Malaquais, Dominique

2002 Architecture, pouvoir et dissidence au Cameroun. Paris, Karthala.

Mauss, Marcel

1936 "Les techniques du corps ", Journal de Psychologie 32 (3-4).

[Réédité in Sociologie et anthropologie, Paris, PUF, 1950.]

Mbembe, Achille

1992 "Provisional Notes on the Postcolony ", Africa 62 (1) : 3-37.

1996 La Naissance du maquis dans le Sud-Cameroun (1920-1960). Paris, Karthala.

Olivier de Sardan, Jean-Pierre

1993 «La surinterprétation politique (les cultes de possession hawka du Niger)", in Jean-François Bayart, ed., Religion et modernité politique en Afrique noire. Paris, Karthala : 163-213.

\section{Parlebas, Pierre}

1999 Jeux, sports et sociétés. Lexique de praxéologie motrice. Paris, INSEP.

Perrot, Claude-Hélène \& François-Xavier Fauvelle-Aymar, eds

2003 Le Retour des rois. Les autorités traditionnelles et l'État en Afrique contemporaine. Paris, Karthala.

Rouch, Jean

1955 Les Maîtres Fous. Film 36 min. Les Films de la Pléiade.

Scott, James C.

1985 Weapons of the Weak. Everyday Forms of Peasant Resistance. New Haven, Yale University Press.

2009 La Domination et les arts de la résistance. Fragments du discours subalterne. Paris, Amsterdam. 
Shaw, Rosalind

2002 Memories of the Slave Trade. Ritual and the Historical Imagination in Sierra Leone. Chicago, University of Chicago Press.

\section{Smith, Pierre}

1984 "Le "mystère" et ses masques chez les Bedik ", L’Homme 24 (3-4) : 5-33.

\section{Stoller, Paul}

1995 Embodying Colonial Memories. Spirit Possession, Power, and the Hauka in West Africa. New York, Routledge.

Tardits, Claude

1960 Contribution à l'étude des populations Bamiléké de l'Ouest Cameroun. Paris, Berger-Levrault.

1980 Le Royaume bamoum. Paris, Armand Colin.

\section{Toulabor, Comi}

1981 «Jeu de mots, jeu de vilains : lexique de la dérision politique au Togo ", Politique africaine 3 : 55-71.
Vansina, Jan

1990 Paths in the Rainforests. Toward a History of Political Tradition in Equatorial

Press.

Warnier, Jean-Pierre

1985 Échanges, développement et hiérarchies dans le Bamenda pré-colonial, Cameroun. Stuttgart, Franz Steiner Verlag Wiesbaden.

1989 "Traite sans raids au Cameroun", Cahiers d'études africaines 29 (113) : 5-32.

1996 "Rebellion, Defection and the Position of Males Cadets : A Neglected Category", in I. Fowler \& D. Zeitlyn, eds, African Crossroads. Intersection Between History and Anthropology in Cameroon. Oxford, Berghahn Books : 115-124.

2007 The Pot-King. The Body and Technologies of Power. Leiden, Brill.

\section{Zempléni, András}

1984 "Secret et sujétion : pourquoi ses "informateurs" parlent-ils à l'ethnologue?", Traverses 30-31: 102-115.

1996 "Savoir taire: du secret et de l'intrusion ethnologique dans la vie des autres ", Gradhiva 20 : 23-41. 